\title{
Anti-glycin-receptor antibody related stiff-person syndrome under treatment with an immune checkpoint inhibitor
}

\author{
Nils Schröter ${ }^{1} \mathbb{D} \cdot$ Cornelius Weiller $^{1} \cdot$ Sebastian Rauer $^{1} \cdot$ Cornelius F. Waller $^{2}$
}

Received: 3 September 2020 / Revised: 20 November 2020 / Accepted: 4 December 2020 / Published online: 24 December 2020

(c) The Author(s) 2020

\section{Dear Sirs,}

We here report the case of a 59-year-old male patient who developed a stiff-person syndrome (SPS) as a possibly immune-related adverse event after receiving Pembrolizumab as first-line therapy of adenocarcinoma of the lung.

In September 2019, a 59-year-old male patient presented to our emergency department with reoccurring painful, immobilizing muscle spasms, stiffness, and falls which had lasted for approximately 10 days. Spasms were triggered by any voluntary movement, sensory, emotional or acoustic stimuli and were located mainly in the legs and the lower back, leading to a lumbar hyperlordosis. The patient reported an improvement of spasm severity and frequency under selfmedication with cannabidiol and baclofen. He suffered from stage IV adenocarcinoma of the lung, treated with the antiPD-1 antibody pembrolizumab. The patient had suffered from pembrolizumab associated increase in transaminases and diarrhea, both grade I, in August 2019 dissolving without further intervention.

On physical examination, the patient presented with hyperreflexia of the lower extremities with spastic tendencies but no weakness or other neurological deficit. Magnet resonance imaging (MRI) of the head and spine were unremarkable. Analysis of cerebrospinal fluid (CSF) revealed mild pleocytosis, $7 / \mu \mathrm{l}(<5)$, and mild disturbances of the blood-brain-barrier with a CSF/serum quotient of albumin of $9.4(<8)$. Initial analysis of paraneoplastic anti-neuronal antibodies and autoimmune encephalitis antibodies,

Nils Schröter

nils.schroeter@uniklinik-freiburg.de

1 Clinic of Neurology and Neurophysiology, Medical Center, Faculty of Medicine, University of Freiburg, Breisacher Str. 64, 79106 Freiburg, Germany

2 Department of Haematology, Oncology and Stem Cell Transplantation, Faculty of Medicine, University Medical Centre Freiburg, University of Freiburg, Freiburg, Germany including anti-GAD, anti-amphysisin, anti-DPPX, and antiGABA-B, were negative in CSF and blood serum, respectively. A secondary analysis revealed anti-glycine receptor antibodies (anti-GlyR). Pathological evaluation and immunophenotypical analysis of cells were unremarkable. Repeated staging by CT-thorax and abdomen did not exhibit new tumor growth. In the synopsis of anamnesis, clinical findings and data of laboratory testing the clinical diagnosis of anti-GlyR related SPS was made.

We initiated therapy with IV immunoglobulin (IVIG) for 5 days and symptomatic therapy with diazepam and morphine stopping symptom progression. Due to reoccurring muscle spasms, the patient was still immobilized and highly impaired in quality of life. In close agreement with the treating oncologists, we followed up IVIG therapy with methylprednisolone $40 \mathrm{mg}$ per day. This led to an improvement in muscle spasm frequency and severity (Fig. 1).

After 4 weeks of rehabilitation, symptoms were alleviated. Even though the patient still suffered from reoccurring muscle spasms and stiffness he was able to walk $500 \mathrm{~m}$ using a rollator. In October 2019, 3 months after the last infusion of pembrolizumab, tumor progress was detected and treatment with pembrolizumab was reinitiated. After a follow up of six months after initial presentation no relapse of SPS occurred and ${ }^{18} \mathrm{~F}$-FDG PET showed stabilization of tumor manifestations.

We present the first case of a stiff-person syndrome as an immune-related adverse event (irAE) under checkpointinhibitors. The diagnosis of anti-GlyR positive SPS was based on the positive antibody results, the classic phenotype comprising co-contraction of agonists and antagonists, rigidity, lumbar hyperlordosis and "startle" induced spasms by tactile, acoustic or emotional triggers [1]. This assumption is supported by the apparent response to immunoglobulins and steroids.

The PD-1 receptor plays a key role in preventing T-cell mediated autoimmune responses [2]. That is why pharmacologically inhibiting the function of the PD-1 pathway 
Fig. 1 Timeline of the case reported. The upper line shows the course of the tumor and the pembrolizumab therapy. The middle row shows the course of causal therapy for stiff-person syndrome. The bottom line shows the course of the symptomatic therapy of the stiff-person syndrome. SPS, stiff-person syndrome; IVIG, intravenous immunoglobulin therapy
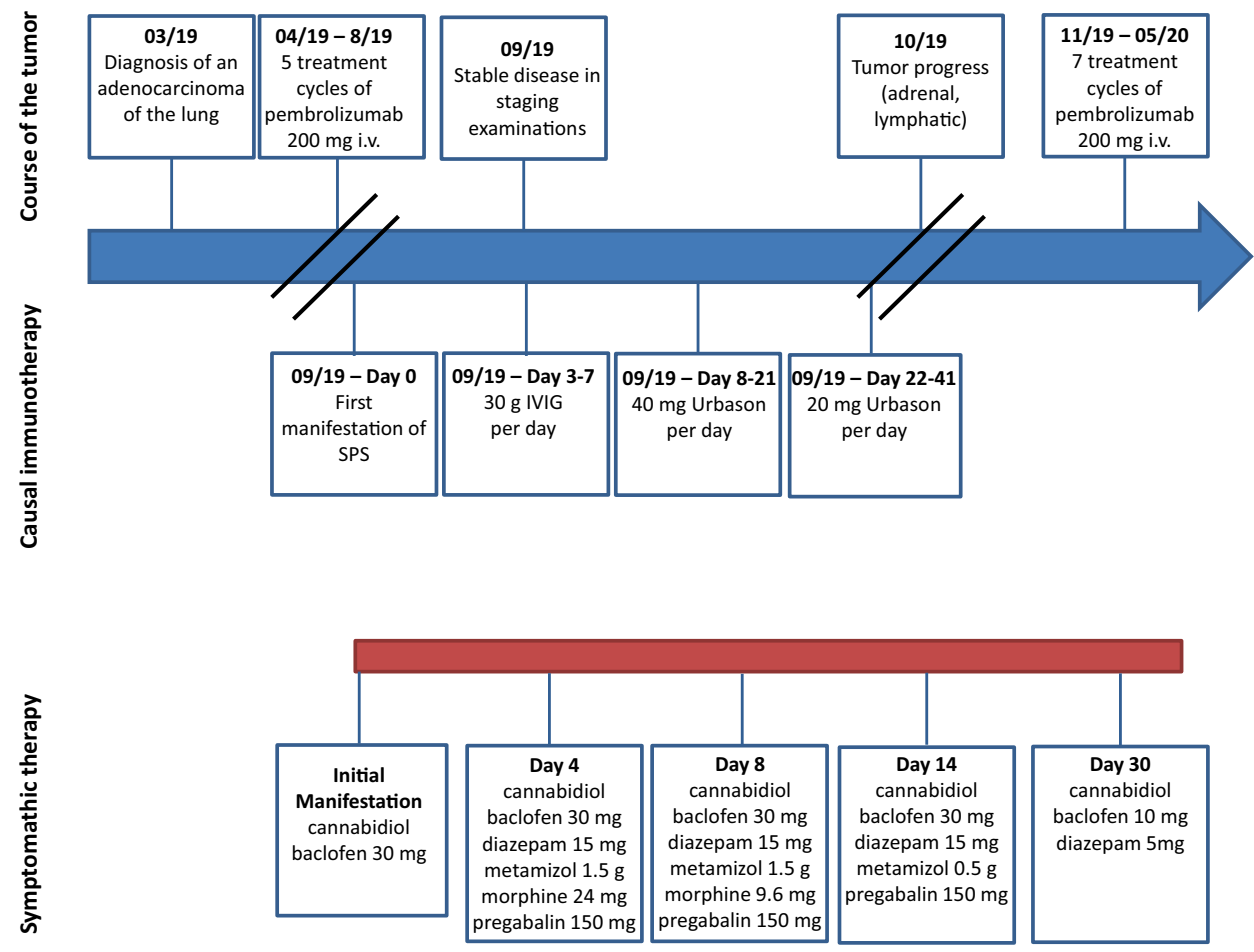

leads on the one hand in PD-1 expressing malignancies to enhanced antitumor immune response and on the other hand, predisposes to irAEs that can occur in up to $27 \%$ of patients. Typically irAEs manifest in the skin, endocrine system, gastrointestinal tract or liver.

SPS can occur as a rare paraneoplastic syndrome in patients without treatment with ICIs [3]. In our view, the lack of tumor progression in the staging examinations and the long latency between tumor growth and the onset of SPS indicates a non-paraneoplastic genesis.

Similar to previous irAEs, SPS remained stable under the continuation of pembrolizumab. This is in line with current ASCO clinical practice guidelines regarding other neurological adverse events, stating that ICIs can be resumed in close consultation with the patient after symptoms have declined [4].

According to the present case report, SPS might be a rare but important irAE checkpoint inhibitors clinicians should be aware of.

Acknowledgements Nils Schröter was funded by the Berta-OttensteinProgramme for Clinician Scientists, Faculty of Medicine, University of Freiburg

Author contributions N.S. conceptualized and drafted the report, C.W., S.R., C.F.W. conceptualized and critically revised the report.
Funding Open Access funding enabled and organized by Projekt DEAL. N.S: Berta-Ottenstein-Programme for Clinician Scientists, Faculty of Medicine, University of Freiburg.

\section{Compliance with ethical standards}

Conflicts of interest N.S., C.W., S.R., C.F.W have nothing to report.

Open Access This article is licensed under a Creative Commons Attribution 4.0 International License, which permits use, sharing, adaptation, distribution and reproduction in any medium or format, as long as you give appropriate credit to the original author(s) and the source, provide a link to the Creative Commons licence, and indicate if changes were made. The images or other third party material in this article are included in the article's Creative Commons licence, unless indicated otherwise in a credit line to the material. If material is not included in the article's Creative Commons licence and your intended use is not permitted by statutory regulation or exceeds the permitted use, you will need to obtain permission directly from the copyright holder. To view a copy of this licence, visit http://creativecommons.org/licenses/by/4.0/.

\section{References}

Baizabal-Carvallo JF, Jankovic J (2015) Stiff-person syndrome: insights into a complex autoimmune disorder. J Neurol Neurosurg Psychiatry [Internet]. 86(8):840-8. Available from: https://jnnp. bmj.com/content $/ 86 / 8 / 840$

Francisco LM, Sage PT, Sharpe AH (2010) The PD-1 pathway in tolerance and autoimmunity. Immunol Rev [Internet]. 236:219. Available from: https://www.ncbi.nlm.nih.gov/pmc/articles/PMC29 19275/ 
El-Abassi R, Soliman MY, Villemarette-Pittman N, England JD (2019) SPS: Understanding the complexity. J Neurol Sci [Internet]. 404:137-49. Available from: http://www.sciencedirect.com/scien ce/article/pii/S0022510X19302813

Brahmer JR, Lacchetti C, Schneider BJ, Atkins MB, Brassil KJ, Caterino JM, et al (2018) Management of immune-related adverse events in patients treated with immune checkpoint inhibitor therapy: American Society of Clinical Oncology Clinical Practice Guideline. J Clin Oncol Off J Am Soc Clin Oncol [Internet]. 36(17):1714-68. Available from: https://www.ncbi.nlm.nih.gov/ pmc/articles/PMC6481621/ 